\title{
« Moy Traductrice » : le façonnement de la figure auctoriale dans le paratexte des traductions de Marie de Gournay
}

JEAN-PHILIPPE BEAULIEU

Université de Montréal

Marie Le Jars de Gournay's prolific textual production has attracted increasing scholarly attention in the last few years. One of the still relatively understudied parts of her oeuvre comprises the five translations from Latin that occupy 274 out of the 995 pages of her 1641 Advis. As Montaigne's "fille d'alliance", she seems to be mainly known for her French versions of the Latin quotations in the Essais; however, her own translations from Ovid's Elegies or Virgil's Aeneid have been largely overlooked, as well as the liminary writings-some of which prove quite substantial, as her De la façon d'escrire de Messieurs l'eminentissime Cardinal $\mathrm{du}$ Perron et Bertaut-where Gournay offers her remarks on the art of translation. In this essay, I wish to focus on these paratextual writings to examine the self-defining strategies employed by Marie de Gournay in order to legitimize her translation activities. Faced with the obstacles caused by "the little credit given to [her] sex", as well as the specific challenges posed by her sources, Gournay interestingly presents her work as composed in "such an unscholarly season" ("une saison si peu studieuse"), that is at a time when the humanist ideals of the sixteenth century were no longer valued. This article seeks to attend to the ethos constructed through the dedications and other liminary pieces in the second book of the Advis, and to examine how Gournay's discursive ethos serves to anticipate and guide the reception of her translations. In this context, paratexts should be viewed, not as mere accessories, but rather as an essential discursive framework for the interpretation of Gournay's translations-it is actually quite clear from the arrangement of the volume that the liminary material was designed as a tight interpretive frame for the translated texts. This study will eventually allow us to determine the place of translation within the range of authorial practices embraced by Marie de Gournay. 
a dernière version du recueil de "Meslanges » que Marie Le Jars de Gournay
(1565-1645) a fait paraître en 1641, sous le titre Les Advis, ou, les presens de la Demoiselle de Gournay, comprend quelques traductions du latin publiées initialement entre 1594 et 1634 : une « oraison » de Tacite, une autre de Salluste, l'épître de Laodamie tirée des Hérö̈des d'Ovide, la deuxième Philippique de Cicéron, de même que des portions des premier, deuxième, quatrième et sixième livres de l'Énéide. Non seulement cet ensemble substantiel assure-t-il à Gournay une place de choix parmi les femmes traductrices de l'Ancien Régime, mais l'étalement temporel de ces publications suggère que, dans la mesure où la pratique de la traduction traverse sa longue activité de lettrée, elle ne saurait se limiter à la fonction pédagogique (et à caractère souvent « privé ») que l'on retrouve évoquée dans certains textes liminaires, comme l'épître à Marguerite de Saluces que Marie de Cotteblanche fait figurer en 1579 avant sa version de trois Dialogos de Pedro Mexía ${ }^{1}$. Chez Marie de Gournay, la traduction est certes une activité de la première heure (le texte qui marque sa venue publique à l'écriture en 1594, le Proumenoir de Monsieur de Montaigne, est ainsi accompagné de traductions de l'Énéide), mais l'intérêt constant qu'elle lui a manifesté tout au long de sa carrière, jusque dans son dernier recueil d'Advis de 1641, montre bien l'importance qu'elle accordait, dans l'esprit de l'humanisme renaissant, à la mise en rapport des anciens et des modernes à travers " une activité vouée [...] à l'enrichissement de la langue $»^{2}$. Encore peu étudiée, la pratique de la traduction par Gournay s'inscrit dans une réflexion plus large sur l'écriture qui a engagé Valerie Worth-Stylianou à constater - avec raison - que cette auteure fait «souvent appel aux mêmes critères pour présenter ses traductions que pour défendre sa conception de la langue française $\star^{3}$, conception radicalement opposée à la réforme malherbienne qui a remodelé le paysage littéraire français au cours des premières décennies du XVII ${ }^{\mathrm{e}}$ siècle ${ }^{4}$.

À l'exception de Sur la version des Poetes antiques, traité sur les métaphores qui se trouve dans le premier livre des Advis, les réflexions traductologiques de Gournay, tout comme ses traductions, sont rassemblées au début du second livre du recueil en un ensemble serré de 275 pages qui témoigne du désir d'encadrer ses versions du latin par des discours préfaciels ou dédicatoires à caractère parfois théorique. Dans une étude antérieure, je me suis penché sur certains des effets occasionnés par une telle dispositio ${ }^{5}$, qui, comme le montre le tableau figurant à la fin de cette étude, souligne le soin avec lequel Gournay a combiné six traductions et sept paratextes en un ensemble 
dont le pivot est constitué par le discours le plus long: De la façon d'escrire de Messieurs l'Eminentissime Cardinal du Perron, et Bertaut Illustrissime Evesque de Sées (désigné comme la pièce 5 dans le tableau), autour duquel se déploient symétriquement deux groupes de six textes. J'aimerais poursuivre ma réflexion sur cet ensemble en me penchant plus particulièrement sur l'ethos auctorial, c'est-à-dire sur l'image d'elle-même qu'y façonne Gournay et qui lui permet de lier théorie et pratique de la traduction à travers la figure de traductrice que donnent à voir les éléments du paratexte ${ }^{6}$. Cet ethos ne constitue pas une donnée uniforme qui serait simplement reprise d'un texte à l'autre : il résulte de la combinaison et de la configuration des modalités éthiques caractérisant certains groupes de ces discours préfaciels. Lorsqu'on les lit en succession, ceux-ci donnent à voir des marques autoréférentielles qui, au bout du compte, projettent l'image d'une femme modulant habilement la rhétorique de la modestie de manière à affirmer la valeur de ses vues et de ses réalisations face tant à ses modèles qu'à ses adversaires, et créant ainsi dans le paratexte un espace où peut résonner sa propre voix ${ }^{7}$, surtout dans les deux discours les plus développés sur lesquels je souhaite mattarder : la Lettre à Monseigneur de Gelas et De la façon d'escrire. La manière dont l'auteure distribue les données relatives à son image semble indiquer une attention portée aux effets syntagmatiques de la dispositio qui n'a probablement rien d'accidentelle lorsqu'on considère le soin avec lequel Gournay a composé et révisé les différentes versions de son recueil ${ }^{8}$. Ainsi, bien qu'elle affiche sa filiation avec les traductions du cardinal Jacques Davy Du Perron (1556-1618) et de Jean Bertaut, évêque de Sées (1552-1611), comme le signale la position centrale du traité De la façon d'escrire, qui donne à voir en clair le nom de ses modèles, Gournay ne le fait pas d'emblée, réservant à ses premiers paratextes, surtout l'épître à Claude de Gelas (pièce 1), le soin d'exposer ses propres vues traductologiques, sans référence à d'autres théoriciens, tout en s'en prenant aux traducteurs qui malmènent la langue française en « travestiss[ant] à la gaillarde mode qui trotte, cette venerable \& majestueuse Antiquité »?

Dès le départ, s'établissent ainsi deux facettes de l'ethos de Gournay comme traductrice : d'une part, l'affirmation primordiale de son statut de théoricienne de la traduction et, plus largement, de poéticienne, associée, d'autre part, à une façon combative d'exposer ses idées et de défendre ses textes au moyen d'une posture d'affrontement marquée qui semble relever de la rhétorique éristique. Gournay fait donc le choix de s'avancer seule, mais déterminée 
à en découdre avec ses adversaires, au seuil de cet ensemble de textes, de même que dans la troisième pièce paratextuelle, l'Advis sur la traduction de la seconde Philippique, où elle dénonce le peu de respect que certains de ses contemporains ont de la culture antique, au contraire d'elle-même qui se révèle sur ce plan l'héritière des idéaux humanistes ${ }^{10}$ :

Mais puisque je tombe sur ce pas, je ne m'en puis sortir sans me pleindre de l'insolence \& de l'erreur d'aucuns Translateurs de ce temps : lesquels outre la licence que je nottois n'agueres, de remaçonner à leur fantaisie, le stile \& les textes pour les enrichir [de] ce [qui] leur semble d'Art \& de bon sens, s'efforcent de baptiser de nos termes $\&$ de depeindre soubs l'image de nos mœurs \& de nos façons de faire [...]. (672-3)

Ce n'est évidemment pas le cas de son travail, dont elle justifie certains choix particuliers, comme d'offrir une version plus longue que le discours de Cicéron, de manière à reconstituer le contexte d'énonciation et les données relatives à l'actio de l'original :

Si moy Traductrice manquant de ces rayons de lumiere [c'est-à-dire les données contextuelles] pour m'exprimer, ne les eusse cherchez à ma necessité dans cette sobre paraphrase ; j'eusse avec peu de peine, faict d'une tres-belle Oraison un tres-impertinent galimathias, ainsi que je voy faire à d'autres assez souvent, en interpretant les bons Livres. (672)

Après cette mise en situation, elle prend la peine de se montrer bien entourée, qu'il s'agisse d'amis (dans la dédicace A Madame de Peray, pièce 2), de protecteurs réels ou potentiels (dans les dédicaces à Louis XIII et au maréchal de Bassompierre, pièces 6 et 7), ou de "parrains » intellectuels, tels Du Perron et Bertaut, dans le noyau que constituent les pièces 5 et 6 . Il me semble que la stratégie de Gournay est de s'appuyer sur ces liens pour affirmer en fin de compte l'intérêt de ses propres réalisations, au moyen d'un jeu de rapprochement qui, parce qu'il maintient une certaine distance avec les figures qu'elle convoque, contribue à la valorisation de l'auteure.

C'est ce que montre, d'entrée de jeu, la façon dont elle utilise le destinataire du premier discours dédicatoire du groupe, Claude de Gelas (1564-1630), évêque d'Agen, comme prétexte pour exposer sa propre conception de la 
traduction. Sous le couvert d'une modestie de bon aloi, Gournay offre à ce prélat la traduction de deux « oraisons » de Tacite et Salluste, «n'agueres traduictes par simple passe-temps » (635), et censément commandée par lui, ce qui représente une façon indirecte de souligner la valeur de son propre travail, puisque Gelas, en tant que traducteur des mêmes textes, a jugé sa version à elle digne d'être publiée. Gournay s'engage par la suite dans la présentation de considérations théoriques sur les difficultés de la traduction, sans lien avec Gelas, sinon que par des interpellations ponctuelles à caractère phatique qui servent à maintenir le contact avec le destinataire. Selon elle, « il faut en traduisant édifier \& recomposer à peu prés les Autheurs de l'Antiquité » (639), reprenant à son compte l'image désormais courante de la traduction (et plus largement de la translatio culturelle) comme activité métaphorique de digestion et d'assimilation $^{11}$. Dans ce contexte, le traducteur doit avoir une « intelligence harmonique \& mystique de ces Escrits-là » et se montrer «sçavant en François, plus qu'il ne l'est en Latin » (637). Cette vision de la traduction comme bien autre chose qu'un simple "labeur de pure peine \& diligence " (636) est portée par l'ethos implicite d'une théoricienne qui se révèle indissociable du rôle de critique de son temps. En effet, l'auteure constate avec indignation l'écart existant entre l'idéal qu'elle fixe et les coutumes de l'époque, se faisant le juge des traducteurs de son temps, peu doués, peu disposés à l'effort et « se traduisant l'un l'autre » :

J'en voy pourtant qui font entendre des Livres qu'ils n'entendent que par auprés : mais ils enjambent effrontement par dessus une difficulté, ou la gauchissent ou l'effleurent simplement sans l'approfondir : se persuadans par un jugement superficiel, que le Lecteur se contentera d'une expression superficielle. (638)

Elle fait appel à des images colorées pour fustiger ses contemporains qui refusent d'utiliser certains synonymes ou métaphores, et qui, se privant ainsi de moyens pour rendre justice à l'original, s'activent en pure perte : "O que de peine prend la guenon à grimper sur un arbre, pour n'y avoir toutesfois autre affaire, que de monstrer son derriere ? » (746). Avec son franc-parler, Gournay s'en prend à ceux qui estiment " qu'une femme ne peut entendre le Latin ». Cette attaque vise entre autres ceux qui lui ont adressé des critiques au sujet de la traduction des citations latines qu'elle a procurées aux éditions de 1617, 1625 et 1635 des Essais de Montaigne : 
Ils me croyent tellement ignorante du Latin, que jusques à ceste immense nuée de passages estallez aux Essais ; \& en la tres-difficile version desquels j'ay rompu la glace ; ils devinent sans prendre la peine de s'en informer, que j'ay desrobé du texte de ce Livre le fil de ma conduicte à mesure que je traduisois. (639-40)

Elle se défend bien de "translater » à partir d'autres traductions, comme certains l'ont laissé entendre en considérant le fait qu'elle a appris par elle-même le latin en "confrontant des Traductions aux Originaux " (639) ${ }^{12}$. La façon autodidacte dont elle dit s'être familiarisée avec le latin - qui n'est guère inattendue pour une femme dans le contexte de la Renaissance ${ }^{13}$ où, comme le rappelle Antoine Berman, "on apprend à écrire en traduisant ${ }^{14}-$ n'a pas grand chose à voir avec ses habitudes de traductrice, selon elle. Si certaines personnes le pensent, c'est que le remaniement de traductions antérieures est désormais chose courante : " [...] c'est un mestier fort vulgaire aujourd'huy, que d'interpreter du François en François » (639), affirme-t-elle. C'est pourquoi elle mentionne nommément les sept traductions de la Philippique qu'elle a consultées (celles d'Antoine Macault (1549), Jean Papon (1554) et d'Antoine de Laval (1586), entre autres ${ }^{15}$ ) afin d'" offrir au curieux, si bon luy semble, le plaisir de la varieté par leur lecture » (674), c'est-à-dire l'occasion de constater qu'elle n'a pas repris servilement ses prédécesseurs dont «l'explication [est] fort souvent differente de la [s]ienne », conformément au principe de la varietas dont elle se fait le chantre ${ }^{16}$.

Comme c'est souvent le cas chez Gournay, la défense de soi est colorée par la question du gender, c'est-à-dire que son appartenance au sexe féminin devient un enjeu indissociable de ceux, plus généraux, des aspects théoriques qu'elle soulève. C'est sur cette question que son discours se personnalise le plus, l'auteure se drapant dans une attitude outragée devant ses contemporains qui font peu de cas des réalisations d'une femme ${ }^{17}:$ «[... je ne puis assez souvent rire, si rire se doibt, de la litterature du Siecle, \& de l'équité dont les femmes sont traictées par telles personnes ", écrit-elle dans la lettre à Mgr de Gelas (640). Avant même leur présentation, les traductions de Gournay se voient annoncées sous un jour polémique, invitant le lecteur à devenir le juge de dernière instance de ses écrits. Pour insister sur cet effet, Gournay a ajouté, à partir de 1626, une adresse au lectorat qui, servant de pont entre les prescriptions de la lettre et les traductions elles-mêmes, illustre bien la façon dont elle assume la 
responsabilité de ses textes, reléguant le dédicataire au second plan pour interpeller directement le lecteur en tant que témoin de son statut de traductologue et de celui de traductrice, l'un renvoyant à l'autre selon une logique qui fait de la pensée de Gournay l'étalon de ses propres réalisations :

Si ceux qui liront les Versions suivantes, y voyent quelque chose qui leur semble de prime abord hardiment traduicte, ou brusquement ; ils sont priez de se souvenir des precautions que j'ay proposées pour la traduction, en la Lettre precedente : \& de considerer en suitte, que j'ay deu prendre pour moy, les conseils que je donne aux autres pour ce regard. $(641)^{18}$

Le texte le plus volumineux du groupe, Sur la façon d'escrire, reprend, en l'élargissant et, surtout, en la détaillant, la conception de la poétique dont Gournay a exposé les grandes lignes dans la Lettre à Mgr de Gelas. Exprimant les doléances de l'auteure contre les "censeurs de son époque ", ce traité de 39 pages, qui, dans le recueil de 1626 publié sous le titre de L'Ombre de la Damoiselle de Gournay, servait initialement de préambule au Bouquet de Pinde et à la Deffence de Poesie, utilise la traduction comme point de départ et d'arrivée du discours, et trouve pour cette raison sa place parmi les traductions, même si le propos de Gournay consiste surtout en des considérations de nature poétique, comme les rimes et les métaphores. Placé sous le parrainage explicite de Du Perron et de Bertaut, le traité fait appel aux deux prélats comme des auctoritates qui confèrent une légitimité au discours de Gournay, mais que celle-ci traite à son propre gré, selon les avenues qu'elle veut donner à sa démonstration et de façon à éclairer ses propres positions, en tant que défenderesse d'une esthétique dont elle apparaît comme le point d'aboutissement : « [...] je declare que je veux escrire, rymer \& raisonner de toute ma puissance, à la mode de Ronsard, Du-Bellay, Des-Portes [...] \& consequemment à la mode aussi des mesmes Prelats, premiers reformateurs de cet Art depuis ces trois fondateurs " (736). Gournay confère auxdits prélats un rôle central, mais instrumental, en faisant bien sentir qu'elle est la maitresse d'œuvre de la démonstration. Sur un ton incisif, Gournay se dit investie d'une mission, à l'instar d'un médecin cherchant à éradiquer une maladie (dans ce cas, les conceptions défavorables à un sain développement de la langue) : 
Quelqu'un me demandoit, si j'entre en cette lice par un espoir que mes remonstrances ou mes exemples, les rappellent. Nullement : suffit que j'advertisse les sains de se garder de la contagion des malades : \& que je face voir, que la Nature m'ayant departy quelque lumiere de raison, je croys estre obligée d'accuser l'erreur où je la trouve : \& de la reprimer selon mon pouvoir, non seulement par mes advis, mais aussi par mes exemples. (736)

Le soupçon de modestie dont elle témoigne n'enlève rien au caractère impérieux de son projet "prophylactique " : il s'agit de dénoncer les « Sectateurs de ce nouvel Art » (738) qui nuisent à la culture française en optant pour la voie de la facilité et de la joliesse :

Ils doivent veritablement [mes avis et exemples], tous feibles qu'ils soient, servir de reproche à tant de gens \& mesmes de condition importante, que je voy chaqu'un jour trahir par leurs Escrits, la Langue de leur Patrie \& leur jugement, soubs une lasche flatterie vers cette Cabale [c'est-à-dire la clique malherbienne]. (736)

En une saison qu'elle juge «si peu studieuse » (734), elle formule son projet, dès la deuxième page, de dénoncer « les ergotteries de ces nouveaux docteurs " pour proposer une poétique plus élevée qu'elle enseignera en décrivant le bon et le mauvais usages du vers :

[...] je dresse en cet Escrit une espece de plan de l'Art legitime de faire des Vers \& du bastard encore, à qui les veut apprendre : j'entends apprendre l'un par suitte, \& l'autre par fuitte : par fuyte, soubs l'image de ces visions nouvelles [les conceptions à la mode]: par suyte, soubs l'exemple des deux Prelats alleguez [...]. (735)

Gournay adopte la position d'un défenseur du bon sens et de la raison ; elle finit par se moquer de ses adversaires qui, dit-elle, " ne meriteroient de nous qu'une risee pour replique »(735), bien que, comme elle l'indique deux pages plus loin, elle ne sache plus si elle doit « rire ou [s] e courroucer sur ce passage ». Pour dévaluer le point de vue de ceux qu'elle ne nomme jamais, en plus d'alléguer des exemples tirés des écrits et traductions des deux prélats, Gournay se cite 
elle-même au moyen de nombreuses autoréférences, parfois générales (« [...] ainsi que j’ai fait voir au Traitté des Rymes »), parfois très précises («Le Lecteur aura peu voir quelques autres de ces exceptions au Traicté des Metaphores, en la Section: Car que logerons nous \& en la Deffense de la Poesie, Traicté second : sur tout en une autre Section qui commence [par] Voyla donc pour conclure " (771)). En plus d'attirer l'attention sur les croisements internes de l'œuvre, afin de souligner la cohérence des préoccupations linguistiques de Gournay qui trouvent dans ce traité un lieu de synthèse, cette façon de faire accentue la visibilité de l'auteure en regard de ses modèles et projette l'image d'une poéticienne aguerrie - en dépit du « peu de credit de [s] on sexe » (734) —, qui n’en est pas à sa première réflexion en la matière.

Comme on l'a déjà senti, un trait qu'accuse parfois le discours préfaciel est l'usage de la rhétorique de la modestie de façon à suggérer une altérité bien assumée par l'auteure. Lorsqu'elle affirme dans l'adresse au cardinal $\mathrm{Du}$ Perron (pièce 6) : « Je sens bien que mes Escrits rudes \& simples se trouvent fort souvent à l'essort en des sentiers de traverse » (773), n'a-t-on pas l'impression qu'un certain sentiment de singularité, voire de fierté, l'emporte sur la valeur dépréciative de l'assertion? Et quand elle confesse à son dédicataire : "Voicy de surcharge une des bisarreries, ou, si l'on veut un des extravagances de mon Livre $[. .$.$] qu'il va fermer sans Eloge cette lettre à une personne de tel merite "$ (774), l'aveu de la distance prise face aux conventions ne lui donne-t-il pas l'occasion d'afficher ses idiosyncrasies? Si elle propose au lecteur de lui « donner courtoisement quelque meilleur conseil » sur les passages difficiles, c'est afin d'inciter celui-ci à juger pour soi de la valeur de ses textes dans un domaine où les comparaisons sont faciles à effectuer.

Elle-même invite d'ailleurs à cette comparaison, car lorsqu'elle s'attaque à ce monument que représente l'Énéide pour les lettrés des XVI et XVII siècles, elle n'hésite pas à se mesurer à ses prédécesseurs immédiats ${ }^{19}$, en présentant ses propres traductions partielles de l'Énéide en contiguïté avec celles de Du Perron et de Bertaut. Ainsi, elle propose une suite de son cru à certains fragments des premier et quatrième livres de l'Énéide traduits par Du Perron, offrant, avant sa propre " continuation ", les vers du cardinal qui lui servent de " joincture " (775). Cette façon de faire relève d'une " hardiesse » (773) qui cherche à souligner sa filiation avec l'éminent homme de lettres, en la situant entre la modestie et la présomption. Gournay procède différemment dans le cas de Bertaut : elle fournit au lecteur côte à côte sa traduction et de celle procurée par Bertaut de 
certaines pièces du deuxième livre, invitant à une "facile confrontation des Traductions faites sur mesme suject » (794). Ce principe de concurrence est annoncé par le Discours sur ce Livre qui ouvre les Advis : « Passons aux Traductions de Virgile, les unes en suite du Cardinal du Perron, les autres en concurrence de Monsieur Bertault, \& que j'avois en ma jeunesse publiées avant luy» $\left(\mathrm{biij}^{\mathrm{vo}}\right)$. Non seulement l'auteure prend-elle ainsi ses distances face à Bertaut - au point même de devoir, dans le Discours sur ce Livre, se défendre d'avoir choisi les " pires eschantillons » du prélat pour « les opposer aux [s]iens [...] par quelque ambition malicieuse » $\left(\mathrm{biij}^{\mathrm{vo}}\right)$, c'est-à-dire pour faire valoir sa version —, mais elle affirme que, par son antériorité, sa " translation » à elle ne saurait être interprétée comme une simple variante des vers de l'évêque de Sées ${ }^{20}$. Au contraire, il s'agit d'une retraduction (c'est-à-dire une nouvelle traduction ${ }^{21}$ ) de l'Énéide, qui, par le dispositif même de présentation des extraits, accentue la rivalité des deux versions et met l'idée de renouvellement qu'implique la retraduction ${ }^{22}$ au service d'une ambition personnelle marquée par l'enjeu de la " généricité ", comme le révèle la dédicace au roi datée de 1626 :

Quelle temerité, SIRE, une quenouille attaque une crosse, \& la crosse illustre d'un Bertault ? duquel à parler serieusement neantmoins, je ne m'approche, qu'affin de porter en reverence le Livre devant luy dans le sainct mystere des Muses : bien qu'il fust raisonnable que soubs un Monarque si magnanime que LOUIs treiziesme, les Dames osassent entreprendre des gestes heroïques. (795)

Teintée par ce qu'elle appelle une « simple rusticité » (795), cette témérité - celle d'une Amazone, comme le précisent les éditions antérieures, où on peut lire l'expression " gestes d'Amazone » au lieu de " gestes héroïques $»^{23}-$ me semble signaler un désir de se distinguer, de se faire une place qui lui est propre, en affichant la marginalité qu'elle dit être la sienne : «Or, SIRE, reduicte à la solitude \& rangée à l'escart, en mon Siecle, je choisis en ce lieu, suivant le train de mon sort, une voye escartée \& sauvage à vous abborder [...]» (795).

Tous ces énoncés paraissent contredire l'opinion de Valerie WorthStylianou qui, à propos de cette mise en rapport des versions, affirme que Gournay « ose - presque par coquetterie - faire paraitre des éléments de leurs traductions à côté de la sienne $»^{24}$. À mon sens, la coquetterie - trait qui n'est guère gournayen - n'a que peu à voir avec l'affaire. Les rapports établis avec les 
modèles, en général, sont plus complexes qu'il n'y parait. Même le lien privilégié avec Montaigne, son "père d'alliance », doit faire l'objet de nuances ${ }^{25}$. Dans le domaine de la traduction qui nous intéresse ici, l'hommage tourne à la rivalité et donne lieu à un concours avec non seulement une crosse, mais bien deux, dont il revient au lecteur de juger les fruits ${ }^{26}$. Curieusement, Valerie WorthStylianou constate, mais sans développer la réflexion, que Gournay " a beau louer les rimes et les archaïsmes de Bertaut, elle ne les imite pas elle-même ${ }^{27}$, puisqu'elle préfère des rythmes plus courts et vigoureux. Manifestement, l'émulation a ses limites ; c'est ce qu'indique clairement la contiguïté matérielle de sa version et de celles de ses deux modèles, dispositif qui révèle en fin de compte autant la différence que la parenté.

Pour présenter son travail de traductrice, Gournay fait ainsi appel à des postures analogues à celles qu'affiche l'ensemble du recueil et qui dénotent un désir de se montrer liée à des modèles et à des protecteurs face à ses adversaires, alliance qui permet de mettre en relief ses aptitudes et réalisations propres. Il en ressort l'image d'un auteur dont le statut de femme, clairement perceptible, semble y être pour quelque chose dans la position - quelque peu isolée et singulière - qu'elle dit être la sienne. Bien mise en évidence dans des textes autoréférentiels comme l'Apologie pour celle qui escrit ${ }^{28}$, cette façon apparemment paradoxale d'être à la fois normative et marginale s'exprime avec vigueur, comme nous l'avons vu, dans le domaine de la traduction, considérée comme un vecteur de développement culturel à travers le dialogue entre passé et présent, entre langue ancienne et langue vernaculaire. On pourrait toutefois affirmer que cette posture de Gournay traverse l'ensemble de sa production, se modulant différemment selon la nature et la matière des discours ; dès 1594, un passage du Proumenoir de M. de Montaigne - que je citerai en guise de conclusion, laissant à Gournay le mot de la fin comme elle l'aurait elle-même voulu - annonce de manière éloquente cette disposition éthique de l'auteure : « Les grands esprits ont tousjours des extravagances hors l'usage, d'autant qu'ils se sont persuadez que ce qui est extravagance à la coustume est reduction à la raison. Et ne faut pas s'estonner si les belles et fortes ames se peuvent si mal assujettir aux regles des entendemens communs $»^{29}$. 


\title{
Tableau
}

\section{Disposition des paratextes et des traductions qui se trouvent au début du second livre des Advis, ou, les Presens de la Demoiselle de Gournay (Paris : J. Du Bray, 1641)}

\section{Discours préfaciel}

(Pièce 1) Lettre à Monseigneur de Gelas, Illustrissime Evesque d'Agen. Sur la version de deux Oraisons Latines (p. 635641) Publiée initialement dans Versions de quelques pieces de Virgile, Tacite et Saluste (F. Bourriquant, 1619).

(Pièce 2) A Madame de Peray Marie de S. Mesmin, sa cousine. Luy dediant l'Epistre de Laodamia, prise d'Ovide (p. 660-662). Dédicace publiée originellement dans L'Ombre de la Damoiselle de Gournay (J. Libert, 1626).

(Pièce 3) Advis sur la traduction de la seconde Philippique de Ciceron (p. 669-674). Publié initialement dans L'Ombre (1626).

(Pièce 4) De la façon d'escrire de Messieurs l'Eminentissime Cardinal $d u$ Perron, et Bertaut Illustrissime Evesque de Sées. Qui sert d'advertissement au Lecteur sur les Poësies de ce Volume: et de suite, encore, à nostre Deffence de la Poësie (p. 733-772). Texte publié pour la première fois dans L'Ombre (1626), mais s'inspirant du Traité sur la Poësie qui figurait dans les Versions de 1619.

\section{Traduction}

Harangue de Galba, traduicte de Tacite (p. 642-647) suivie de la Harangue de Marius au Peuple Romain, traduicte de Saluste (p. 648-659). Publiée d'abord dans Versions de quelques pieces [...] (1619).

Epistre de Laodamia à Protesilaus. Version d'Ovide (p. 663-669). Publiée d'abord dans L'Ombre (1626).

Version de la deuxiesme Philippique de Ciceron. (p. 676-732). Publiée initialement dans L'Ombre (1626).

\footnotetext{
(1)
} 
(Pièce 5) A Monseigneur l'Eminentissime Cardinal du Perron (p. 773-774). Texte publié dans L'Ombre (1626).

(Pièce 6) Au Roy (p. 794-796). Adresse publiée initialement dans Versions (1619).
Partie du Premier de l'Æneide, commençant où l'Eminentissime Cardinal $d u$ Perron acheve de le traduire. (p. 775793). Publiée dans les Eschantillons de Virgile (T. du Bray, 1620 et 1623). La traduction de Du Perron provient de Partie du premier et quatriesme livre de l'Æneide (R. Estienne, 1611).

Version du Second de l'Eneide. (p. 797835) (en alternance avec des fragments de la traduction de Bertaut). Publiée initialement et intégralement dans Le Proumenoir de Monsieur de Montaigne (A. L'Angelier, 1594). Gournay cite la traduction de Bertaut qui figure dans le Recueil des ceuvres poétiques (A. L'Angelier, 1605).

(Pièce 7) A Monseigneur le Mareschal de Bassompierre (p. 836-837). Texte publié dans L'Ombre (1626).
Partie du Quatriesme de l'Æneide, commençans comme dessus apres le mesme Cardinal (p. 838-865 ; publiée dans les Traductions (1621)), suivie de la Version du Sixiesme de l'Eneide (p. 866-909), publiée dans les Advis (T. Du Bray, 1634).

\section{Notes}

1. Anne R. Larsen, « Marie de Cotteblanche : Préfacière et traductrice de trois dialogues de Pierre Messie », Études littéraires 27.2 (1994), p. 114-116.

2. Valerie Worth-Stylianou, «Marie de Gournay et la traduction : défense et illustration d'un style », in Marie de Gournay et l'édition de 1595 des Essais de Montaigne, Actes du Colloque organisé par la Société Internationale des Amis de Montaigne (9 et 10 juin 1995), éd. Jean-Claude Arnould (Paris : Champion, 1996), p. 199. 
3. Valerie Worth-Stylianou, "Marie de Gournay traductrice ", et "Introduction ", in Euvres complètes de Marie de Gournay, dir. Jean-Claude Arnould (Paris : Champion, 2002), p. 58.

4. Gournay a mené son combat contre cette nouvelle esthétique parallèlement à Jean-Pierre Camus et à François La Mothe Le Vayer. À ce sujet, consulter Anna Lia Franchetti, L'Ombre discourante de Marie de Gournay (Paris : Champion, 2006), p. 75. Voir aussi Dudley Wilson, « Ronsard, Mlle de Gournay and the Poetic and Linguistic Crisis of the Late Sixteenth and Early Seventeenth Centuries ", in Mélanges sur la littérature de la Renaissance à la mémoire de V.-L. Saulnier, éd. P.-G. Castex (Genève : Droz, 1984), p. 427-436.

5. Jean-Philippe Beaulieu, " $\grave{A}$ la charnière de la traduction et du "discours de raison" : l'épître dédicatoire chez Marie de Gournay ", in La rhétorique épistolaire sous l'Ancien Régime. De la théorie aux pratiques, éd. Claude La Charité, Collection République des Lettres (Québec : Presses de l'Université Laval, à paraître).

6. Depuis quelques années, diverses études ont amorcé une réflexion sur l'importance de l'ethos dans la mise en place textuelle de l'image de Gournay. Voir notamment Jean-Philippe Beaulieu, « Marie de Gournay ou l'occultation d'une figure auctoriale ", Renaissance et Réforme/Renaissance and Reformation 24.2 (2000), p. 23-34, de même que "Feminine Authorial Ethos : The Use of Marie de Gournay's Discours sur ce livre as an Introduction to Her Collected Works ", in Teaching French Women Writers of the Renaissance and Reformation, éd. Colette H. Winn (New York: The Modern Language Association, 2011), p. 170-178. On consultera également le chapitre « Marie de Gournay : construction d'un personnage et d'une personne ", de la biographie signée par Michèle Fogel, Marie de Gournay. Itinéraires d'une femme savante (Paris : Fayard, 2004), p. 19-39.

7. On comprendra ainsi la nécessité de citer abondamment Gournay dans le cadre d'une étude portant sur l'ethos, précisément pour faire sentir les inflexions de sa voix et la coloration qu'elle donne à ses propos.

8. À ce sujet, on consultera le chapitre intitulé « L'ombre et le monument : l'œuvre en chantier » qu'Anna Lia Franchetti (L'Ombre discourante, p. 15-38) consacre à Gournay comme éditrice de ses propres textes. Voir également Jean-Philippe Beaulieu, "Relecture et réécriture dans les recueils de Marie de Gournay ", in Lectrices d'Ancien Régime, éd. Isabelle Brouard-Arends (Rennes : Presses Universitaires de Rennes, 2003), p. 243-251.

9. Marie de Gournay, Les Advis, ou, les Presens de la Demoiselle de Gournay (Paris : Jean Du Bray, 1641), p. 671. Désormais, tous les renvois à ce recueil figureront 
entre parenthèses après la citation. Le lecteur peut consulter l'édition des Euvres complètes de Marie de Gournay (dir. Jean-Claude Arnould) qui reproduit le texte et la pagination des Advis de 1641.

10. Giovanna Devincenzo, Marie de Gournay. Un cas littéraire (Fasano, Paris : Schena Editore, Presses de l’Université de Paris-Sorbonne, 2002), p. 97.

11. Formulée entre autres par Joachim Du Bellay. Voir sa "préface » à La Deffence, et illustration de la langue françoyse (1549), éd. Jean-Charles Montferran (Genève : Droz, 2001), p. 24-25.

12. L’Apologie pour celle qui escrit, qui termine le premier livre des Advis, contient également cette indication sur l'apprentissage du latin (605bis), que Gournay utilise probablement pour souligner l'écart entre sa formation et ses réalisations.

13. Au sujet de l'éducation de femmes à la fin de la Renaissance, voir Linda Timmermans, L'accès des femmes à la culture (1598-1714) : un débat d'idées de saint François de Sales à la marquise de Lambert (Paris : Champion, 1993), p. 45 et suiv.

14. Antoine Berman, « De la translation à la traduction », TTR : traduction, terminologie, rédaction 1.1 (1988), p. 24.

15. Au sujet de ces traductions, voir Claude Longeon, Une province française à la Renaissance (Saint-Étienne : Centre d'Études Foréziennes, 1975), p. 279-280.

16. Elle écrit à ce sujet : «[...] l'entendement humain \& la faculté d'interpretation, à peu pres comme celle de l'invention, sont des outils vastes : \& la fluste de Pallas avoit plusieurs pertuys, comme la lyre d'Apollon, plusieurs cordes à divers tons » (674).

17. Ce qui ne l'empêche pas de parfois critiquer le rôle des femmes de son époque dans l'évolution de la langue ; Cathleen M. Bauschatz, " Marie de Gournay and the Crisis of Humanism ", in Humanism in Crisis. The Decline of the French Renaissance, éd. Philippe Desan (Ann Arbor : The University of Michigan Press, 1991), p. 289-290.

18. Lorsqu'il annonce les traductions du deuxième livre des Advis, le Discours sur ce Livre qui sert de préambule à l'ensemble du recueil, reprend la même idée en l'associant à une invitation à comparer ses versions à celles de ses prédécesseurs : «Aux Versions qui la suivent, il est requis d'observer en premier lieu, si je suy les reigles que je prescrits moy-mesme en cette Lettre [à Gelas]. Cela consiste en deux poincts principaux, la vigueur \& la fidelité de l'expression, \& apres, la grace \& l'addresse. Tous ces deux poincts s'observeront par eux-mesmes \& par la confrontation d'autres Versions qui se sont faites par le passé sur les mesmes Pieces que je traduis » $\left(\right.$ biij $\left.^{\mathrm{vo}}\right)$. 
19. Depuis Octovien de Saint-Gelais (1509), plusieurs lettrés ont traduit des parties de l'Énéide, notamment Louis Des Masures (1547), Joachin du Bellay (1552), Jacques Peletier du Mans (1580), Antoine et Robert Le Chevalier d'Agneaux (1582), Jean Bertaut (1605), Jacques Davy Du Perron (1610) ; voir Bruno Méniel, Renaissance de l'épopée. La poésie épique en France de 1572 à 1623 (Genève : Droz, 2004), p. 45.

20. Sur Bertaut traducteur de l'Énéide, voir Georges Grente, Jean Bertaut, abbé d'Aunay, premier aumônier de la reine, évêque de Séez, 1552-1611 (Genève : Slatkine Reprints, 1969 [1903]), p. 270 et suiv.

21. À propos de la notion de retraduction, on consultera l'exposé synthétique que fournit Marie-Alice Belle dans «Sur la retranscription de Virgile en Angleterre au XVII ${ }^{\mathrm{e}}$ siècle : les enjeux politiques et esthétiques de l'Énéide de John Ogilby (1654)», Études Epistémè 12 (2007), p. 51-52. (http://www.etudes-episteme.org/ ee/articles.php?lng=fr\&pg=344) Site consulté le 4 juin 2012.

22. Antoine Berman, «La retraduction comme espace de la traduction", Palimpsestes 4 (1990), p. 1 ; Annie Brisset, "Retraduire ou le corps changeant de la connaissance ", Palimpsestes 15 (2004), p. 48-49 et 55-56.

23. Voir le commentaire de Michèle Fogel, Marie de Gournay. Itinéraires d'une femme savante, p. 232-233, sur ce passage.

24. Valerie Worth-Stylianou, «Introduction » aux Euvres complètes, p. 61.

25. Le signataire de cet article est de ceux qui estiment que Gournay, tout en revendiquant son lien avec Montaigne, a progressivement cherché à en estomper certaines traces. Voir Jean-Philippe Beaulieu, «Relire Marie de Gournay, sans Montaigne? », in Terrae Incognitae de l'écriture féminine, éd. Marie-Laure Girou Swiderski (New York, Ottawa : Legas, 2004), p. 73-83.

26. Dans une étude consacrée aux traductions parues dans le Proumenoir, Martine Debaisieux constate un désir « [d]'émancipation de l'auteur vis-à-vis de la tradition » représentée par les figures paternelles que sont Montaigne et les deux prélats déjà mentionnés : Martine Debaisieux, « Marie de Gournay cont(r)e la tradition : Du Proumenoir de Monsieur de Montaigne aux versions de l'Énéide ", Renaissance et Réforme/Renaissance and Reformation, 31.4 (1997), p. 46 et 52.

27. Valerie Worth-Stylianou, "Introduction ", Euvres complètes, p. 63.

28. Au sujet de la rhétorique propre à ce texte, voir Sylvie Gourde, « Écriture contre parole. Marie de Gournay et son autodéfense dans Apologie pour celle qui escrit », Tangence 77 (2005), p. 61-72.

29. Marie de Gournay, Euvres complètes, p. 1357. 